\title{
Fraturas atípicas do fêmur associadas ao uso crônico de bisfosfonatos: Uma série de casos de 66 pacientes*
}

\section{Atypical femur fractures associated with chronic bisphosphonates use: A case series with 66 patients}

\author{
Guilherme Guadagnini Falotico ${ }^{1}$ J) Jose Fernando Flores Cunza ${ }^{10}$ Gabriel Ferraz Ferreira1(1)

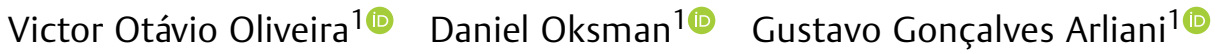

\footnotetext{
${ }^{1}$ Departamento de Ortopedia e Traumatologia, Instituto Prevent
} \\ Senior, São Paulo, SP, Brasil \\ Endereço para correspondência Gabriel Ferraz Ferreira, Rua Cerro \\ Corá, 585, Sala 605, Torre 1, São Paulo, SP, Brasil \\ (e-mail: gabriel.ferraz38@yahoo.com.br).
}

Rev Bras Ortop 2022;57(5):851-855.

\section{Resumo \\ Palavras-chave \\ - fraturas do fêmur \\ - bisfosfonato \\ - osteoporose \\ Objetivo Descrever os resultados de uma série de pacientes tratados cirurgicamente com diagnóstico de fratura femoral atípica associada ao uso de bisfosfonatos, assim como correlacionar o tempo de uso prévio da medicação com o tempo de consolidação da fratura e comparar o tempo de consolidação das fraturas completas e incompletas. Métodos Trata-se de um estudo observacional e retrospectivo de 66 pacientes com diagnóstico de fratura atípica do fêmur associada ao uso crônico de bisfosfonatos. Os pacientes foram submetidos ao tratamento cirúrgico ortopédico em hospital de referência no período de janeiro de 2018 a março de 2020. \\ Resultados Os pacientes incluídos no estudo eram todos do sexo feminino, com dois casos bilaterais. A consolidação da fratura ocorreu em todos os casos com tempo médio de 2,3 meses e seguimento de 5,8 meses. O tempo médio de uso de bisfosfonatos foi de 7,8 anos. Não houve correlação do tempo de uso prévio de bisfosfonatos com o tempo de consolidação das fraturas. Houve uma diferença do tempo de consolidação entre as fraturas completas e incompletas. \\ Conclusão Houve consolidação após tratamento cirúrgico com haste cefalomedular longa em todos os pacientes do presente estudo, sendo o tempo de consolidação maior nas fraturas completas em relação às incompletas, e não houve correlação entre o tempo de uso prévio de bisfosfonatos e o tempo de consolidação. \\ Nível de evidência Nível IV, série de casos}

Trabalho desenvolvido no Departamento de Ortopedia e Traumatologia, Instituto Prevent Senior, São Paulo, SP, Brasil.

recebido

16 de Setembro de 2020

aceito

01 de Dezembro de 2020

Publicado on-line

Março 31, 2021
DOI https://doi.org/ 10.1055/s-0041-1726066. ISSN 0102-3616. (c) 2021. Sociedade Brasileira de Ortopedia e Traumatologia. All rights reserved.

This is an open access article published by Thieme under the terms of the Creative Commons Attribution-NonDerivative-NonCommercial-License, permitting copying and reproduction so long as the original work is given appropriate credit. Contents may not be used for commercial purposes, or adapted, remixed, transformed or built upon. (https://creativecommons.org/ licenses/by-nc-nd/4.0/)

Thieme Revinter Publicações Ltda., Rua do Matoso 170, Rio de Janeiro, RJ, CEP 20270-135, Brazil 


\begin{abstract}
Keywords

- femoral fractures

- bisphosphonate

- osteoporosis

Objective The present study aims to describe outcomes from a series of surgically treated patients with atypical femoral fracture due to bisphosphonates use, in addition to correlate the time of previous medication use with fracture consolidation time, and to compare the consolidation time of complete and incomplete fractures.

Methods This is an observational, retrospective study with 66 patients diagnosed with atypical femur fractures associated with chronic bisphosphonates use. The patients underwent orthopedic surgical treatment at a referral hospital from January 2018 to March 2020.

Results All patients were females, with two bilateral cases. Fracture consolidation occurred in all cases, with an average time of 2.3 months and a follow-up time of 5.8 months. The average time of bisphosphonates use was 7.8 years. There was no correlation between the time of previous bisphosphonates use and the time for fracture consolidation. Consolidation time differed in complete and incomplete fractures.

Conclusion Surgical treatment with a long cephalomedullary nail resulted in consolidation in all patients. The consolidation time was longer in complete fractures when compared with incomplete lesions, and there was no correlation between the time of previous bisphosphonates use and the consolidation time .

Level of evidence Level IV, case series
\end{abstract}

\section{Introdução}

A osteoporose é definida como a redução gradual e progressiva da massa óssea. Os fatores de risco principais para sua ocorrência são: sedentarismo, alcoolismo, tabagismo, uso prolongado de corticosteroides e a redução da biodisponibilidade de cálcio e vitamina D. ${ }^{1}$ As fraturas com maior associação à osteoporose são de: quadril, coluna vertebral, extremidade distal do rádio e extremidade proximal do úmero. $^{2}$ Neste cenário, o tratamento medicamentoso da osteoporose é recomendado como prevenção primária destas fraturas e a droga de primeira escolha, em geral, são os bifosfonatos. $^{3}$

$\mathrm{O}$ uso prolongado de bisfosfonatos pode levar à perda progressiva da propriedade elástica dos ossos, ${ }^{4}$ o que representa fator de risco para a ocorrência de fraturas femorais atípicas, definidas como fraturas que ocorrem entre $o$ trocânter menor e a região supracondiliana do fêmur (subtrocantérica ou diáfise), incompletas (acometendo isoladamente a cortical lateral) ou completas de padrão transverso, após trauma mínimo. ${ }^{5-8}$

O risco de fratura atípica aumenta para indivíduos que usam bisfosfonatos orais por $>3$ anos (tratamento médio de 7 anos), sendo que as mulheres têm um risco absoluto 3 vezes maior que os homens, e o alendronato é a droga aventada como de maior risco para fraturas atípicas. ${ }^{5}$

A literatura descreve bons resultados no tratamento destas fraturas com uso de hastes intramedulares, ${ }^{9}$ porém o tempo de consolidação em geral é bastante prolongado, o que pode ser explicado pela ação dos bisfosfonatos no calo mole, suprimindo sua remodelação. ${ }^{10}$

O objetivo do presente estudo é descrever os resultados de uma série de pacientes tratados cirurgicamente com diag- nóstico de fratura femoral atípica associada ao uso de bisfosfonatos, bem como correlacionar o tempo de uso prévio da medicação com o tempo de consolidação da fratura e comparar o tempo de consolidação das fraturas completas e incompletas.

\section{Material e métodos}

Trata-se de um estudo observacional e retrospectivo de 66 pacientes ( 2 casos bilaterais), totalizando 68 fraturas atípicas do fêmur associadas ao uso crônico de bisfosfonatos. Os pacientes foram submetidos ao tratamento cirúrgico ortopédico em hospital de referência no período de janeiro de 2018 a março de 2020.

\section{Critérios de inclusão e exclusão}

Os pacientes incluídos no estudo atenderam aos seguintes critérios: 1) fraturas de fêmur atípicas completas e incompletas em pacientes sintomáticos; 2) pacientes com uso crônico ( $>5$ anos) de bisfosfonatos; e 3) idade $>60$ anos.

Já os critérios de exclusão foram: 1) pacientes com diagnóstico de infecção osteoarticular ipsilateral do quadril atual ou prévia; 2) fraturas osteoporóticas típicas do fêmur (colo do fêmur, transtrocanterianas, subtrocantéricas); e 3 ) fraturas patológicas associadas a tumores primários ou metastáticos e outros distúrbios ósseos (por exemplo, doença de Paget ou displasia fibrosa).

\section{Coleta dos dados, avaliação radiográfica e técnica cirúrgica}

Os dados foram coletados diretamente do prontuário eletrônico médico hospitalar, após a aprovação do comitê de ética. As variáveis incluídas foram gênero, idade, índice de massa 


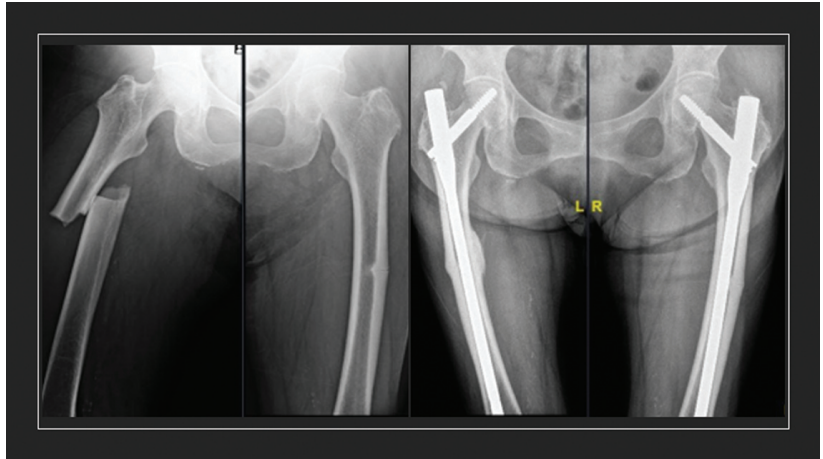

Fig. 1 (A) Imagem radiográfica pré-operatória de fratura atípica bilateral. (B) Imagem radiográfica com 7 meses de pós-operatório.

corpórea (IMC) e tempo de uso prévio contínuo de bisfosfonatos.

As imagens radiográficas foram realizadas de forma padronizada em aparelho digital com ampliação pré-definida de $100 \%$. A análise das imagens foi feita digitalmente pelo software Centricity Universal Viewer Zero Footprint (GE Healthcare, Barrington, IL, EUA). A consolidação foi definida como a formação de calos de ponte em pelo menos três corticais em duas projeções radiográficas (anteroposterior e perfil) por meio do escore Reliability of the Radiographic Union Scale in Tibial Fractures (RUST, na sigla em inglês), expandido para a consolidação de fraturas femorais. ${ }^{11}$

A ausência de sinais radiográficos de consolidação, após $\geq$ 6 meses de pós-operatório, foi definida como pseudoartrose. Todas as avaliações radiográficas foram feitas por 2 ortopedistas com $>5$ anos de experiência, de modo independente. Em caso de eventual falta de consenso dos avaliadores, o pesquisador sênior do estudo foi acionado para a decisão final.

O tratamento cirúrgico com redução fechada em mesa ortopédica foi utilizado para todos os pacientes, com uso de haste cefalomedular longa com dispositivo antirrotatório do parafuso cefálico e utilização de um parafuso de bloqueio distal (-Figura $\mathbf{1}$ ).

O pós-operatório foi padronizado, sendo liberada a carga parcial com andador conforme tolerado desde $01^{\circ}$ dia de pós-operatório, e os pacientes seguiram na fisioterapia motora até a consolidação da fratura. O bisfosfonato foi suspenso após a fratura, sendo indicado o uso de teriparatida em dose de $20 \mathrm{mcg}$ por via subcutânea em dose diária durante 2 anos, conforme protocolo institucional. O seguimento foi realizado em conjunto com o ambulatório especializado em metabolismo ósseo.

\section{Análise estatística}

As variáveis categóricas foram mensuradas pela sua proporção, e as contínuas pelo cálculo da média e do desvio padrão (DP). Utilizou-se o teste de Wilcoxon para a comparação das variáveis contínuas não paramétricas, após a avaliação da distribuição pelo teste de Shapiro. Toda a avaliação estatística foi realizada pelo software $\mathrm{R}$ ( $\mathrm{R}$ Foundation, Viena, Áustria), ${ }^{12}$ por meio do pacote Stats, ambos de fonte aberta. A correlação de variáveis se deu pelo teste de Pearson.

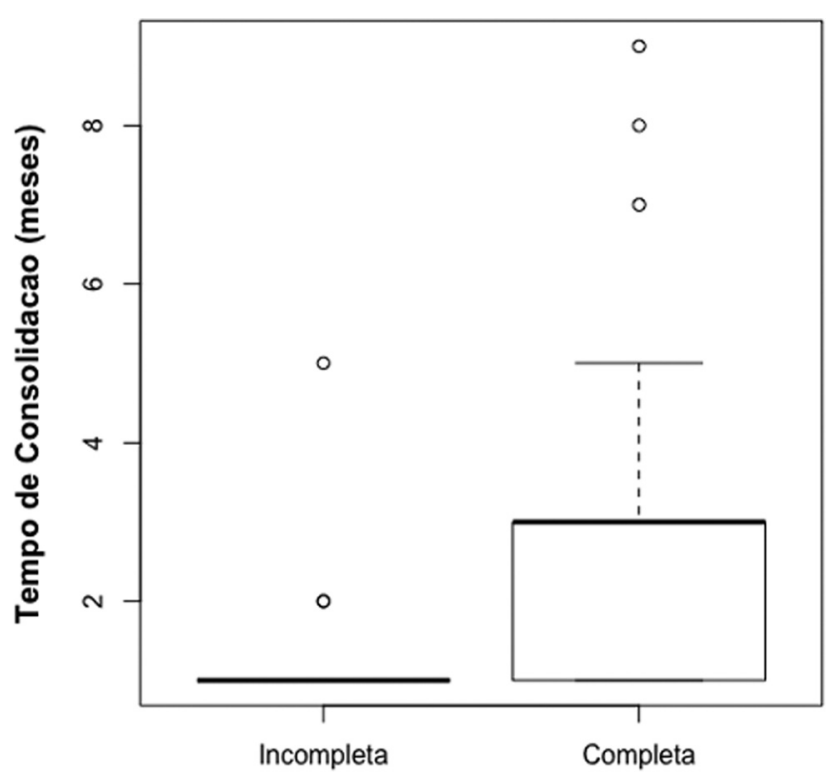

Fig. 2 Distribuição do tempo de consolidação das fraturas em meses relacionado ao tipo de traço completo ou incompleto.

\section{Resultados}

Os pacientes incluídos no presente estudo foram todos do sexo feminino, sendo dois casos bilaterais. As pacientes foram seguidas ambulatorialmente até a consolidação da fratura, que ocorreu em todas as pacientes. O acompanhamento das pacientes apresentou média de 5,8 meses, sendo a média de idade de 78,4 anos, com DP de 6,5, e a maioria das fraturas ocorreu no lado esquerdo (56,5\%). O IMC médio foi de 26,6, com DP de 2,4.

Em relação à localização das fraturas, $65 \%$ foram diafisárias e $45 \%$ na região subtrocantérica do fêmur. Já sobre o tipo anatômico de fratura, houve 55 casos de fraturas atípicas completas $(81 \%)$, das quais a mais comum foi a transversa, seguida pelas fraturas oblíquas e um caso com fratura do tipo cunha simples. A amostra apresentou 13 casos de fraturas atípicas com traço incompleto, representando $\sim 19 \%$.

A consolidação da fratura na análise de radiografia pelo escore de RUST foi de 2,3 meses, com DP de 1,6. Em relação ao tipo de fratura, aquelas com traço incompleto consolidaram em uma média de 1,4 meses, enquanto os traços completos se consolidaram em uma média de 2,5 meses, sendo essa diferença estatisticamente significativa $(p<0,05)$, conforme demonstrado na - Figura 2.

Não foi evidenciada correlação do tempo de uso prévio de bisfosfonatos com o tempo de consolidação das fraturas, por meio do teste de Pearson, com índice de correlação de 0,05 e $p=0,63$.

O espessamento da cortical foi identificado em todos os casos. $\mathrm{O}$ uso de bisfosfonatos teve a sua maioria com utilização do alendronato (84,0\%), seguido pelo risendronato $(8,7 \%)$, ibandronato $(4,3 \%)$, e pamidronato e denosumabe, com $1,5 \%$ cada.

O mecanismo do trauma foi a queda da própria altura na maioria (82,6\%) dos casos, sendo o restante não relacionado com história de trauma. Apenas $26 \%$ das pacientes incluídas 
no estudo referiram dor prévia pregressa à fratura. 0 tempo médio de uso de bisfosfonatos foi de 7,8 anos, com DP de 5,7 anos.

\section{Discussão}

O principal achado do presente estudo é que não houve correlação entre o tempo de uso prévio de bisfosfonatos e o tempo de consolidação da fratura tratada com haste cefalomedular longa. Houve diferença estatisticamente significativa no tempo de consolidação comparando as fraturas completas e incompletas.

A fixação intramedular tem vantagens biomecânicas e biológicas sobre a osteossíntese com placas na fixação de fraturas atípicas. ${ }^{13}$ Em um estudo comparativo entre fraturas convencionais e atípicas, as fraturas atípicas apresentaram taxas mais altas de falha com o uso de placa (30\%) em comparação com fraturas típicas ( $0 \%)$. Já nos pacientes tratados com haste intramedular, a taxa de consolidação foi de $98 \%$; porém, assim como no presente estudo, com um longo tempo de consolidação (8,3 meses).

Já Weil et al. ${ }^{14}$ mostraram que 7 (46\%) das 17 fraturas tratadas com haste intramedular longa fresada precisaram de cirurgia de revisão. Eles atribuíram a alta taxa de falha à consolidação óssea prejudicada pela terapia prolongada com bisfosfonatos, e não à técnica cirúrgica. Além disso, observaram diferenças no tempo de consolidação entre fraturas femorais atípicas reduzidas anatomicamente e não anatomicamente; fraturas reduzidas anatomicamente consolidaram em média 3,7 meses mais rápido do que aquelas fixadas em varo.

As fraturas femorais atípicas incompletas representam um desafio diagnóstico, visto que os pacientes podem apenas apresentar desconforto na coxa ao deambular. Assim, frequentemente, o diagnóstico pode ser feito somente no cenário de uma fratura completa com grande limitação funcional associada. Recomenda-se a realização de uma radiografia simples do fêmur contralateral em pacientes com fratura completa. ${ }^{15-17}$ Consequentemente, a fixação profilática em pacientes com dor persistente pode ser recomendada.

No entanto, a decisão de proceder à cirurgia profilática para uma fratura incompleta deve depender de vários fatores, incluindo a presença de fratura incompleta bilateral, dor persistente, e uma fratura completa no lado oposto. Se um paciente é assintomático, a cirurgia profilática pode não ser justificada. ${ }^{18}$ No presente estudo, as fraturas incompletas, nos pacientes sintomáticos, foram fixadas cirurgicamente.

Quanto ao tempo de consolidação na comparação entre fraturas completas e incompletas submetidas ao tratamento cirúrgico, a literatura carece de evidências definitivas. Alguns estudos citam tempo prolongado de consolidação nas fraturas incompletas tratadas de forma não operatória, bem como em fraturas completas submetidas ao tratamento operatório, o que os autores atribuem, além do efeito biológico do bisfosfonato, ao fato de que em alguns casos houve redução com pequena diástase no foco da fratura. ${ }^{17,19,20}$
A idade média descrita na literatura dos pacientes portadores das fraturas atípicas do fêmur é de $\sim 75$ anos, ${ }^{6}$ assim como foi verificado no presente estudo. Porém, apesar de acometer pacientes idosos, as fraturas atípicas do fêmur não estão associadas ao aumento da mortalidade. ${ }^{7}$

No tratamento medicamentoso do paciente diagnosticado com fratura femoral atípica, os bisfosfonatos devem ser descontinuados. 4,5,15,21 Além disso, há evidências de que agentes antiosteoporóticos com potentes efeitos de formação óssea, como ranelato de estrôncio e, particularmente, a teriparatida, podem melhorar a renovação óssea e a microarquitetura. ${ }^{22,23}$ Todos os pacientes do presente estudo tiveram a interrupção no uso do bisfosfonato e a prescrição da teriparatida após o diagnóstico.

O presente estudo apresenta algumas limitações. Por ser retrospectivo, sugere possível viés de informação quanto à obtenção dos dados do prontuário médico. Além disso, a metodologia empregada em grupo único não permite a verificação estatística de fatores de risco relacionados ao tipo de fratura estudada. Como perspectiva futura de estudo, os autores planejam avaliar os fatores de risco em um desenho de caso-controle, bem como estabelecer um protocolo para o seguimento prospectivo dos pacientes com fraturas atípicas.

\section{Conclusão}

Houve consolidação, após tratamento cirúrgico com haste cefalomedular longa, em todas as pacientes do presente estudo. O tempo de consolidação foi maior nas fraturas completas em relação às incompletas. Não houve correlação entre o tempo de uso prévio de bisfosfonatos e o tempo de consolidação das fraturas femorais atípicas.

\section{Conflito de interesses}

Os autores declaram não haver conflito de interesses.

\section{Referências}

1 Assessment of fracture risk and its application to screening for postmenopausal osteoporosis. Report of a WHO Study Group. World Health Organ Tech Rep Ser 1994;843:1-129

2 Cummings SR, Melton LJ. Epidemiology and outcomes of osteoporotic fractures. Lancet 2002;359(9319):1761-1767

3 Wells GA, Cranney A, Peterson J, et al. Alendronate for the primary and secondary prevention of osteoporotic fractures in postmenopausal women. Cochrane Database Syst Rev 2008;(01): CD001155

4 Odvina CV, Zerwekh JE, Rao DS, Maalouf N, Gottschalk FA, Pak CY. Severely suppressed bone turnover: a potential complication of alendronate therapy. J Clin Endocrinol Metab 2005;90(03): 1294-1301

5 Shane E, Burr D, Abrahamsen B, et al. Atypical subtrochanteric and diaphyseal femoral fractures: second report of a task force of the American Society for Bone and Mineral Research.J Bone Miner Res 2014;29(01):1-23

6 Schilcher J, Michaëlsson K, Aspenberg P. Bisphosphonate use and atypical fractures of the femoral shaft. N Engl J Med 2011;364 (18):1728-1737

7 Russell RG. Bisphosphonates: the first 40 years. Bone 2011;49 (01):2-19 
8 Dell RM, Adams AL, Greene DF, et al. Incidence of atypical nontraumatic diaphyseal fractures of the femur. J Bone Miner Res 2012;27(12):2544-2550

9 Giannoudis PV, Ahmad MA, Mineo GV, Tosounidis TI, Calori GM, Kanakaris NK. Subtrochanteric fracture non-unions with implant failure managed with the "Diamond" concept. Injury 2013;44 (Suppl 1):S76-S81

10 Perren SM. Evolution of the internal fixation of long bone fractures. The scientific basis of biological internal fixation: choosing a new balance between stability and biology. J Bone Joint Surg Br 2002;84(08):1093-1110

11 Azevedo Filho FA, Cotias RB, Azi ML, Teixeira AA. Reliability of the radiographic union scale in tibial fractures (RUST). Rev Bras Ortop 2016;52(01):35-39

12 R: A language and environment for statistical computing. [computer program]. Vienna, Austria: R Foundation for Statistical Computing; 2013

13 Savaridas T, Wallace RJ, Salter DM, Simpson AH. Do bisphosphonates inhibit direct fracture healing?: A laboratory investigation using an animal model Bone Joint J 2013;95-B(09): 1263-1268

14 Weil YA, Rivkin G, Safran O, Liebergall M, Foldes AJ. The outcome of surgically treated femur fractures associated with long-term bisphosphonate use. J Trauma 2011;71(01):186-190

15 Das De S, Setiobudi T, Shen L, Das De S. A rational approach to management of alendronate-related subtrochanteric fractures. J Bone Joint Surg Br 2010;92(05):679-686
16 Capeci CM, Tejwani NC. Bilateral low-energy simultaneous or sequential femoral fractures in patients on long-term alendronate therapy. J Bone Joint Surg Am 2009;91(11):2556-2561

17 Thompson RN, Phillips JR, McCauley SH, Elliott JR, Moran CG. Atypical femoral fractures and bisphosphonate treatment: experience in two large United Kingdom teaching hospitals. J Bone Joint Surg Br 2012;94(03):385-390

18 Wang K, Moaveni A, Dowrick A, Liew S. Alendronate-associated femoral insufficiency fractures and femoral stress reactions. J Orthop Surg (Hong Kong) 2011;19(01):89-92

19 Lee YK, Lee YJ, Lee NK, Nho JH, Koo KH. Low Positive Predictive Value of Bone Scan to Predict Impending Complete Fracture among Incomplete Atypical Femoral Fracture. J Korean Med Sci 2018;33(22):e157

20 Gustafsson A, Schilcher J, Grassi L, Aspenberg P, Isaksson H. Strains caused by daily loading might be responsible for delayed healing of an incomplete atypical femoral fracture. Bone 2016; 88:125-130

21 Papakostidis C, Psyllakis I, Vardakas D, Grestas A, Giannoudis PV. Femoral-shaft fractures and nonunions treated with intramedullary nails: the role of dynamisation. Injury 2011;42(11):1353-1361

22 Goh SK, Yang KY, Koh JS, et al. Subtrochanteric insufficiency fractures in patients on alendronate therapy: a caution. J Bone Joint Surg Br 2007;89(03):349-353

23 Peichl P, Holzer LA, Maier R, Holzer G. Parathyroid hormone 1-84 accelerates fracture-healing in pubic bones of elderly osteoporotic women. J Bone Joint Surg Am 2011;93(17):1583-1587 